\title{
A New 4D Piecewise Linear Multiscroll Chaotic System with Multistability and Its FPGA-Based Implementation
}

\author{
Faqiang Wang $(\mathbb{D}$, Hongbo Cao $\mathbb{i}$, and Dingding Zhai $\mathbb{D}$ \\ State Key Laboratory of Electrical Insulation and Power Equipment, School of Electrical Engineering, Xi'an Jiaotong University, \\ Xi'an 710049, China \\ Correspondence should be addressed to Faqiang Wang; faqwang@xjtu.edu.cn
}

Received 12 February 2021; Revised 20 March 2021; Accepted 19 April 2021; Published 7 May 2021

Academic Editor: Eric Campos

Copyright ( $\odot 2021$ Faqiang Wang et al. This is an open access article distributed under the Creative Commons Attribution License, which permits unrestricted use, distribution, and reproduction in any medium, provided the original work is properly cited.

Due to the complex behavior of a multiscroll chaotic system, it is a good candidate for the secure communications. In this paper, by adding an additional variable to the modified Lorenz-type system, a new chaotic system that includes only linear and piecewise items but can generate $4 n+4$ scroll chaotic attractors via choosing the various values of natural number $n$ is proposed. Its dynamics including bifurcation, multistability, and symmetric coexisting attractors, as well as various chaotic and periodic behaviors, are analyzed by means of attraction basin, bifurcation diagram, dynamic map, phase portrait, Lyapunov exponent spectrum, and $C_{0}$ complexity in detail. The mechanism of the occurrence for generating multiscroll chaotic attractors is presented. Finally, this multiscroll chaotic system is implemented by using the Altera Cyclone IV EP4CE10F17C8 FPGA. It is found that this FPGA-based design has an advantage of requiring less resources for $0 \%$ of the embedded multipliers and $0 \%$ of the PLLs of this FPGA are occupied.

\section{Introduction}

As indicated in many open literatures, the chaotic system that can generate multishape chaotic attractors has complex dynamical behaviors so that it is difficult to decode its information when it is used in the field of secure communications. Hence, exploring the multishape chaotic system and analyzing its complex dynamical behaviors through theoretical analysis, numerical simulations, implementations, and applications is a hot topic all the time, and many researchers exert much effort to investigate this research area. To date, many kinds of multiscroll chaotic systems have been presented [1-7]. For example, according to Chua's circuit and using the sine function, a multiscroll chaotic system was introduced and implemented by an electronic circuit, which consists of the commercial trigonometric function chip AD639 and the corresponding auxiliary chips and basic circuit elements [1]. Based on the cellular neural networks and using the trigonometric function, a multiscroll chaotic system was given and analyzed in [2]. By using the hyperbolic tangent function series as the unique nonlinear function, a multiscroll chaotic system was presented and confirmed by an electronic circuit which is constructed by a unity gain voltage buffer, a single current-feedback operational amplifier, and a transconductor in [3]. In [4], the theories, methods, and applications of generating multiscroll chaotic attractors were summarized. Based on the saturated function series, a multiscroll chaotic system that can generate $1 D n$-scroll, $2 D n \times m$-grid scroll, and $3 D n \times m \times l$-grid scroll chaotic attractors was presented and implemented by using an electronic circuit in [8]. By using the Chen chaotic system and the sine function, a multiscroll chaotic and hyperchaotic system was given in [9]. An improved highorder Chua's circuit that can generate multiscroll chaotic attractor by introducing the signum function series was presented and analyzed in [10]. Also, multiwing chaotic systems that can generate multiwing chaotic attractors have also been concerned intensively and many good topologies were presented, such as multiwing Lorenz chaotic system [11], grid multiwing chaotic system [12], 3D grid multiwing chaotic system [13], 2N-butterfly wing chaotic system [14], and fractional-order multiwing chaotic system [15]. All these 
achievements enrich the chaos theory and the nonlinear circuit and precede their potential application in practical engineering.

In addition, because of the applications of chaotic system in digital secure communication, the realization of chaotic system via digital signal processing circuit attracts people's attention. For example, the multiscroll chaotic systems were implemented in DSP [16], FPGA [17, 18], ARM [19, 20], and Arduino [21, 22]. Generally speaking, as indicated in [23], the implementation based on FPGA has more merits including more flexibility and lower cost. Therefore, in this paper, based on the modified Lorenz-type chaotic system that includes only linear and piecewise items, a new $4 D$ piecewise linear chaotic system that can generate $4 n+4$ scroll chaotic attractors is proposed and analyzed. By employing attraction basin, bifurcation diagram, dynamic map, phase portrait, Lyapunov exponent spectrum, and $C_{0}$ complexity, its dynamical behaviors including multistability, bifurcation, and chaotic and period behaviors are investigated. Also, the mechanism of generating multiscroll chaotic attractors is described theoretically. It is found that symmetrical coexisting attractors will appear in the system with respect to different initial conditions. In addition, the multiscroll chaotic signal generator is implemented by using the digital chip FPGA. Notably, since $0 \%$ of the embedded multipliers and $0 \%$ of the PLLs of the Altera Cyclone IV EP4CE10F17C8 FPGA are occupied in the realization of the proposed chaotic generator, the new multiscroll chaotic system has the advantage of low FPGA resource required.

The paper is organized as follows. In Section 2, the mathematical model of $4 D$ linear piecewise chaotic system is presented. In Section 3, the dynamical behaviors of the proposed chaotic system are analyzed. In Section 4, the mechanism of generating multiscroll chaotic attractors is presented and some numerical simulations are provided. The FPGA implementation of the proposed multiscroll chaotic system is shown in Section 5. Finally, some concluding remarks are presented in Section 6.

\section{A New 4D Piecewise Linear Chaotic System}

In [24], based on the traditional Lorenz system, the modified $3 D$ Lorenz-type chaotic system that includes only linear and piecewise items was introduced. Its differential equations are

$$
\left\{\begin{array}{l}
\dot{x}=a(y-x) \\
\dot{y}_{\mp}=\mp \operatorname{sgn}(x) z \\
\dot{z}_{ \pm}= \pm|x| \mp b
\end{array}\right.
$$

where $a$ and $b$ are positive constant parameters and $x, y$, and $z$ are variables. Here, by adding an additional variable $w$ into equation (1), the following new $4 D$ system that includes only linear and piecewise items can be obtained.

$$
\left\{\begin{array}{l}
\dot{x}=a(y-x) \\
\dot{y}_{\mp}=\mp 16 \operatorname{sgn}(4 x) z \pm 16 \operatorname{sgn}(4 y) w \\
\dot{z}_{ \pm}= \pm 16|x| \mp b \\
\dot{w}=-c w+f(z)
\end{array}\right.
$$

where

$$
f(z)=\sum_{k=-n}^{n} \operatorname{sgn}(4 z+0.5 k),
$$

where sgn () is the signum function, $a, b$, and $c$ are positive constant parameters, $x, y$, and $z$ and $w$ are variables, and $n$ is a natural number.

Taking $n=0$, obviously, there are two modes in system (2). One mode is $\dot{y}=\dot{y}-$ and $\dot{z}=\dot{z}_{+}$which is denoted by $M(-$, $+)$, and the other mode is $\dot{y}=\dot{y}+$ and $\dot{z}=\dot{z}$ - which is denoted by $\mathrm{M}(+,-)$. For $f(z)=\operatorname{sgn}(4 z)$, its value depends on the sign of $z$. If $z>0, f(z)=1$. If $z<0, f(z)=-1$. However, if $z=0, f$ $(z)=0$. It is the same for sgn $(4 x)$ and sgn $(4 y)$.

Thus, from system (2), one can obtain that this chaotic system has the six following equilibrium points:

$$
[X, Y, Z, W]=\left\{\begin{array}{l}
S_{+1,+2}=(( \pm b / 16),( \pm b / 16),(1 / c),(1 / c)), \quad \text { with } z>0, \\
S_{+0,-0}=(( \pm b / 16),( \pm b / 16), 0,0), \quad \text { with } z=0, \\
S_{-1,-2}=(( \pm b / 16),( \pm b / 16),(-1 / c),(-1 / c)) \text { with } z<0
\end{array}\right.
$$

where $X, Y, Z$, and $W$ are denoted by the equilibrium points of system (2). The Jacobian matrix of system (2) at the equilibrium points is

$$
J=\left[\begin{array}{cccc}
-a & a & 0 & 0 \\
\mp 128 \delta(4 X) Z & \pm 128 \delta(4 Y) W & \mp 16 \operatorname{sgn}(4 X) & \pm 16 \operatorname{sgn}(4 Y) \\
\pm 16 \operatorname{sgn}(X) & 0 & 0 & 0 \\
0 & 0 & 8 \delta(4 Z) & -c
\end{array}\right]
$$

where $\delta()$ is the delta function and it is the differential of sgn (). For $\delta(4 Z)$, its value equals zero for all $Z$, except $Z=0$ where it is infinite. When $Z=0$, the related equilibrium points are $S_{+0,-0}$. Because the value of $\delta(0)$ equals infinity, the corresponding eigenvalues of equation (5) cannot be calculated. However, we can obtain the characteristic equation of the Jacobian matrix (5) at $S_{+0,-0}$. For example, choosing $a=32, b=2.4$, and $c=16$, the characteristic equation of equation (5) at $S_{+0,-0}$ is

$$
\lambda^{4}+48 \lambda^{3}+512 \lambda^{2}+8192 \lambda-\inf =0,
$$

where "inf" is positive infinite. Then, the Routh array can be derived to know the roots' characteristics of equation (6) for 
judging the stability of system (2) under the above parameters. Its Routh array is

\begin{tabular}{c|ccc}
$s^{4}$ & 1 & 512 & -inf \\
$s^{3}$ & 48 & 8192 & \\
$s^{2}$ & 341.3 & - inf & \\
$s^{1}$ & $8192+0.14 \times$ inf & \\
$s^{0}$ & - inf &
\end{tabular}

Therefore, based on the Routh-Hurwitz criterion, for the characteristic equation of the Jacobian matrix (5) at $S_{+0,-0}$, it has three roots with negative real part and one root with positive real number, since the number of changes in sign of the first column of the Routh array is one. Hence, these two equilibrium points $\left(S_{+0,-0}\right)$ are unstable point so that the system's trajectory will be departed rapidly at $S_{+0,-0}$.

However, for $S_{+1,+2}$ and $S_{-1,-2}$, even if the Jacobian matrices of system (2) under two modes are different from each other, the characteristic equation of the Jacobian matrix (5) in both two modes is identical and given by

$$
(\lambda+c)\left(\lambda^{3}+a \lambda^{2}+256 a\right)=0 .
$$

Thus, under these four equilibrium points $\left(S_{+1,+2}\right.$ and $S_{-1,-2}$ ), the sets of eigenvalues are the same. Based on the above parameters, the eigenvalues of the Jacobian matrix (5) of the system under two modes is $(-37.7489$, $2.8744+14.4482 \mathrm{i}, 2.8744-14.4482 \mathrm{i},-16)$ which means that all these four equilibrium points are index-2 saddle foci. Thus, the system's trajectory will be in a spiral movement at these four equilibrium points which result in four-scroll attractors. For example, under the initial condition $\left(x_{0}, y_{0}, z_{0}\right.$, $\left.w_{0}\right)=(0.01,0.01,0.01,0.01)$, the time-domain waveforms, the phase portraits, and the Poincaré maps from the numerical simulations by using the Matlab software for system (2) under two modes which are shown in Figures 1 and 2, respectively, can be obtained.

Figures 1(a) and 1(b) show the phase portrait of system (2) under $\mathrm{M}(-,+)$ and Figures 2(a) and 2(b) show its phase portrait under $M(+,-)$. Figures $1(\mathrm{c})$ and 2(c) show the respective time series and Figures 1(d) and 2(d) show the corresponding Poincaré maps. Moreover, based on the Wolf method $[25,26]$, by using the ode 45 algorithm and choosing the simulation time $T=1000 \mathrm{~s}$ with time step being $0.002 \mathrm{~s}$ in Matlab software, the calculated Lyapunov exponents of system (2) under $\mathrm{M}(-,+)$ are $L E_{1}=2.88, L E_{2}=0$, $L E_{3}=-12.15$, and $L E_{4}=-38.43$, and the calculated results under $\mathrm{M}(+,-)$ are $L E_{1}=2.95, L E_{2}=0, L E_{3}=-12.22$, and $L E_{4}=-38.44$. From phase portraits, time series, Poincaré maps in Figures 1 and 2, and the Lyapunov exponents, one can determine that system (2) in both two modes under the above given parameters and initial conditions is in chaotic operation and it has four-scroll chaotic attractors. Note that, because $M(+,-)$ is similar to $M(-,+)$, only $M(-,+)$ is investigated in the following sections.

\section{Dynamical Behaviors of the New Chaotic System}

3.1. Chaotic Attractors for Various Parameters. Suppose that the parameters $b=2.4, c=16, n=0$, and $\left(x_{0}, y_{0}, z_{0}\right.$, $\left.w_{0}\right)=(0.01,0.01,0.01,0.01)$ are fixed and parameter $a$ is changed from 11.2 to 171.2. The Lyapunov exponent spectrum and their enlargement are shown in Figures 3(a) and $3(\mathrm{~b})$, respectively. One can see that system (2) is always in chaotic operation when $a$ is within $(11.2,171.2)$, since $L E_{1}$ is bigger than zero. Figure 4 (a) shows the bifurcation diagram about parameter $a$ versus $y$ under the initial condition $\left(x_{0}, y_{0}, z_{0}, w_{0}\right)=(0.01,0.01,0.01,0.01)$ (blue case) and $\left(x_{0}, y_{0}, z_{0}, w_{0}\right)=(0.01,-0.01,-0.01,0.01)$ (red case). From Figure 4, some typical phase portraits can be obtained.

Case 1. The red color and the blue color in Figure 4(a) are mixed in both positive and negative of $y$. That is to say, the four-scroll chaotic attractors under two types of initial conditions will appear. For example, let $a=19.2$; the four chaotic attractors are shown in Figure 4(b).

Case 2. In Figure 4(a), the red color is only filled in the positive of $y$, while the blue color is only filled in the negative of $y$. Hence, under the initial condition $\left(x_{0}, y_{0}, z_{0}\right.$, $\left.w_{0}\right)=(0.01,-0.01,-0.01,0.01)$, it has two-scroll chaotic attractors in the positive of $y$. However, under the initial condition $\left(x_{0}, y_{0}, z_{0}, w_{0}\right)=(0.01,0.01,0.01,0.01)$, only twoscroll chaotic attractors will appear in the negative of $y$. For example, choose $a=40$; the numerical simulations are shown in Figure 4(c).

Case 3. Both red and blue colors in Figure 4(a) are filled in the negative of $y$. Hence, under the initial condition $\left(x_{0}\right.$, $\left.y_{0}, z_{0}, w_{0}\right)=(0.01,-0.01,-0.01,0.01)$ and $\left(x_{0}, y_{0}, z_{0}\right.$, $\left.w_{0}\right)=(0.01,0.01,0.01,0.01)$, only two-scroll chaotic attractors in the negative of $y$ will appear. For example, take $a=48$; the two-scroll chaotic attractors are shown in Figure 4(d).

Case 4. Both the red color and the blue color in Figure 4(a) are filled in the positive of $y$. Hence, under the initial condition $\left(x_{0}, y_{0}, z_{0}, w_{0}\right)=(0.01,-0.01,-0.01,0.01)$ and $\left(x_{0}\right.$, $\left.y_{0}, z_{0}, w_{0}\right)=(0.01,0.01,0.01,0.01)$, only two-scroll chaotic attractors in the positive of $y$ will appear. For example, choose $a=60.8$; the two-scroll chaotic attractors are shown in Figure 4(e).

Case 5. In Figure 4(a), the red color is only filled in the negative of $y$, while the blue color is only in the positive of $y$. Hence, under the initial condition $\left(x_{0}, y_{0}, z_{0}, w_{0}\right)=(0.01$, $-0.01,-0.01,0.01)$, it has two-scroll chaotic attractors in the negative of $y$. But, under the initial condition $\left(x_{0}, y_{0}, z_{0}\right.$, $\left.w_{0}\right)=(0.01,0.01,0.01,0.01)$, it has two-scroll chaotic attractors in the positive of $y$. For example, choose $a=112$; the numerical simulations are shown in Figure 4(f). 


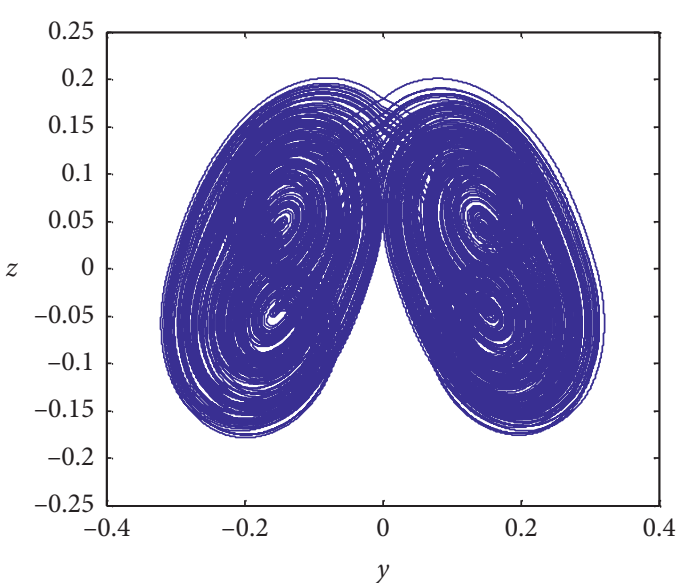

(a)
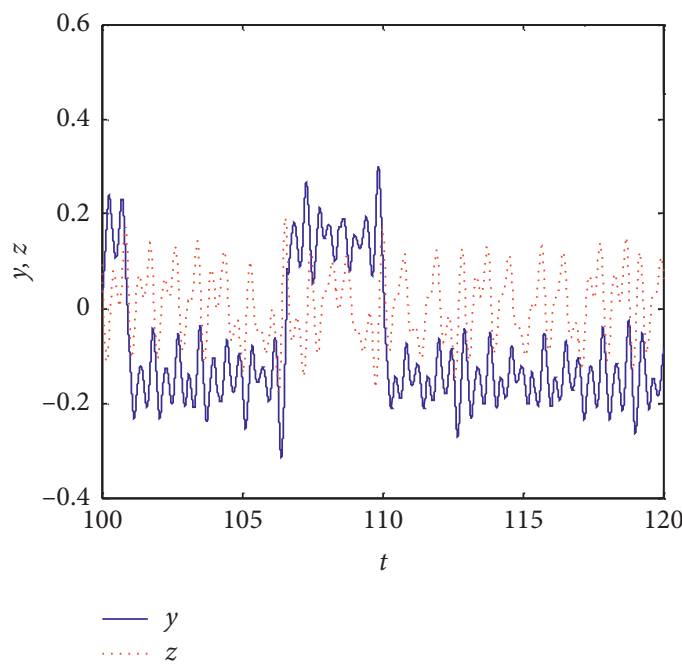

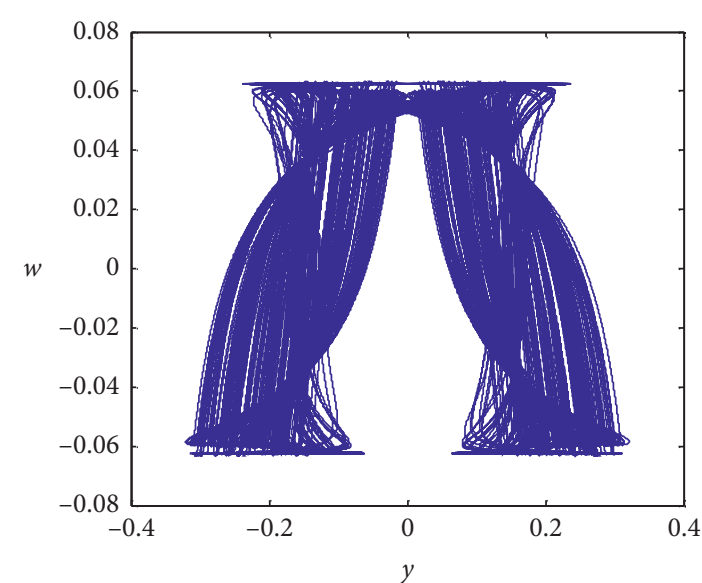

(b)

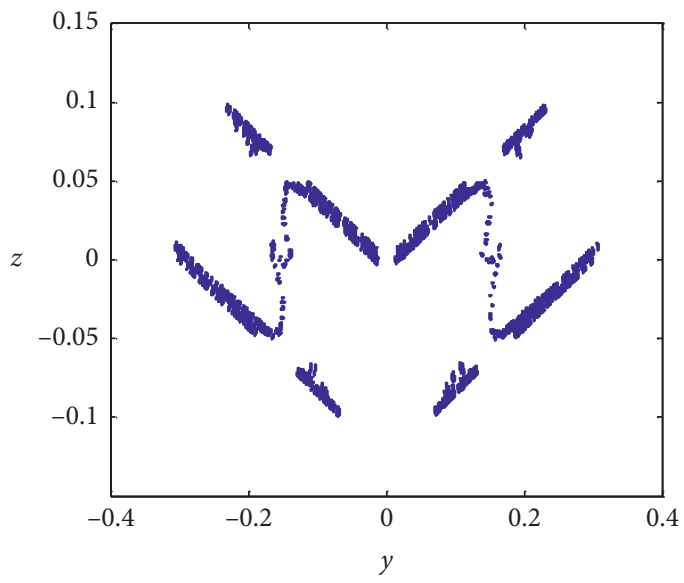

(c)

(d)

Figure 1: Numerical simulations for the proposed multiscroll chaotic system under mode $1: M(-,+$ ). (a) $y$ versus $z$, (b) $y$ versus $w$, (c) $t$ versus $y$ and $t$ versus $z$, and (d) Poincaré map in $y$ versus $z$ under $x=y$.

Note that the influence of parameters $b$ and $c$ on the dynamical behaviors can also be analyzed by using the above technique. Here, under the initial condition $\left(x_{0}, y_{0}, z_{0}\right.$, $\left.w_{0}\right)=(0.01,0.01,0.01,0.01)$ (blue case) and $\left(x_{0}, y_{0}, z_{0}\right.$, $\left.w_{0}\right)=(0.01,-0.01,-0.01,0.01)$ (red case), only the bifurcation diagram about the variation of parameters $b$ and $c$ is presented and shown in Figure 5.

3.2. $C_{0}$ Complexity Analysis on System Parameters. As indicated in [27], $C_{0}$ complexity is defined as the proportion of irregular components in the sequence, and $C_{0}$ algorithm based on fast Fourier transform removes the regular part of the signal transformation domain and leaves the irregular part. The larger the proportion of the irregular part in the sequence is, the closer the corresponding time series is to the random, and the greater the complexity is. Hence, $C_{0}$ algorithm can be used to calculate the randomness of chaotic time series; that is to say, it can be adopted to describe the complexity of chaotic time series [28].
When the length of chaotic time series of the new system is selected as $N=8000$ and the complex parameter is selected as $r=10$, also $C_{0}$ algorithm is used to calculate the complexity of $x$ sequence with respect to varying parameter $a$, as shown in Figure 6(a). From Figures 6(a) and $3(a)$, one can obtain that a large $C_{0}$ complexity value corresponds to a positive Lyapunov exponent, indicating that the complexity of $x$ sequence is high. Comparing Figure 6(a) with Figure $4(\mathrm{a})$, the $C_{0}$ complexity curve has good consistency with its bifurcation diagram. Similarly, the $C_{0}$ complexity of $z$ sequence with respect to varying parameter $b$ is shown in Figure 6(b), and the $C_{0}$ complexity of $w$ sequence with respect to varying parameter $c$ is shown in Figure 6(c). By combining Figures 6(b) and 5(a), as well as Figures 6(c) and 5(b), one can see that the $C_{0}$ complexity of the proposed system in the chaotic state is greater than that of the proposed system in its other states.

Hence, from $C_{0}$ complexity, it can effectively distinguish the dynamic characteristics of continuous chaotic time series 


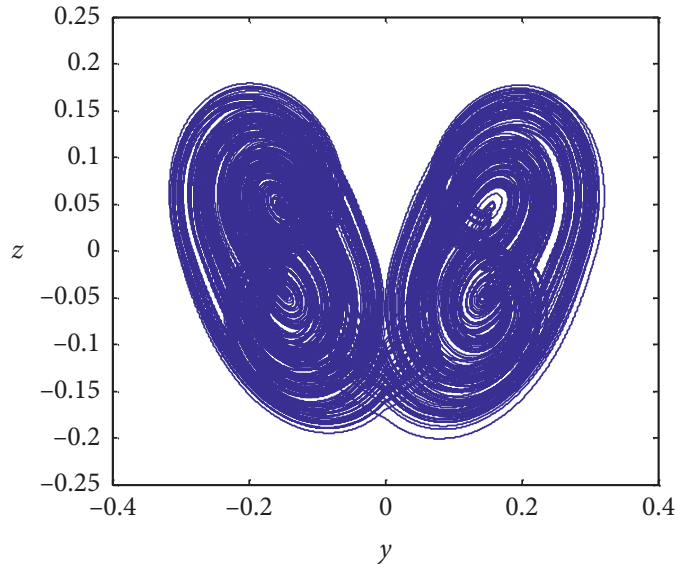

(a)

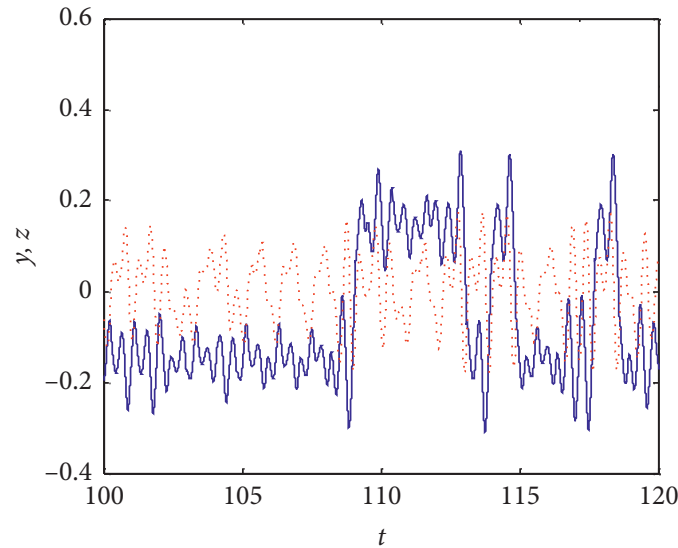

$\begin{array}{ll}- & y \\ \ldots \ldots & z\end{array}$

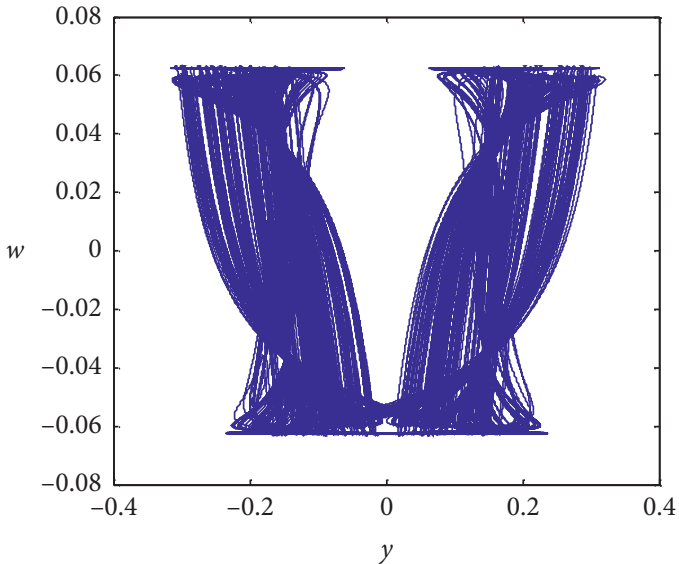

(b)

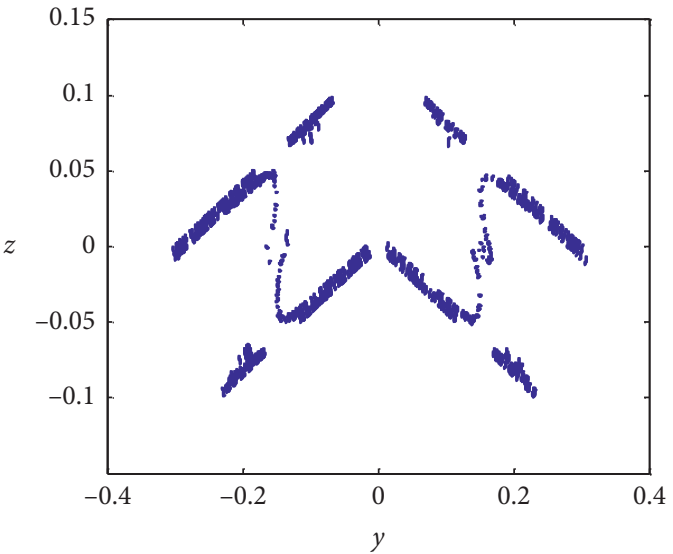

(c)

(d)

Figure 2: Numerical simulations for the proposed multiscroll chaotic system under mode 2: $M(-,+$ ). (a) $y$ versus $z$, (b) $y$ versus $w$, (c) $t$ versus $y$ and $t$ versus $z$, and (d) Poincaré map in $y$ versus $z$ under $x=y$.

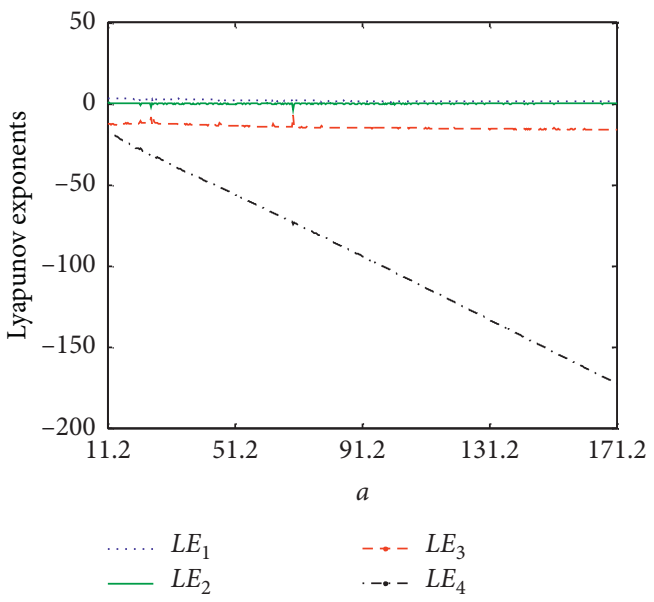

(a)

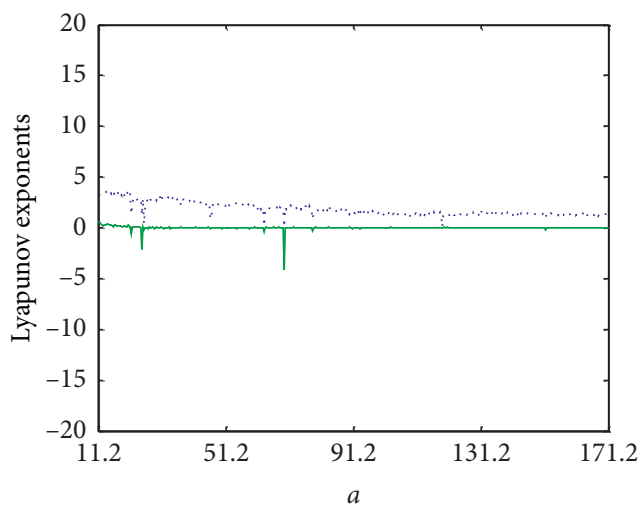

$\begin{array}{ll}\ldots . . . & L E_{1} \\ - & L E_{2}\end{array}$

(b)

FiguRE 3: Lyapunov exponents for the proposed multiscroll chaotic system under $\mathrm{M}(-,+)$ with variation of $\alpha$. (a) $L E_{1}, L E_{2}, L E_{3}$, and $L E_{4}$. (b) Closed-up view of $L E_{1}$ and $L E_{2}$. 


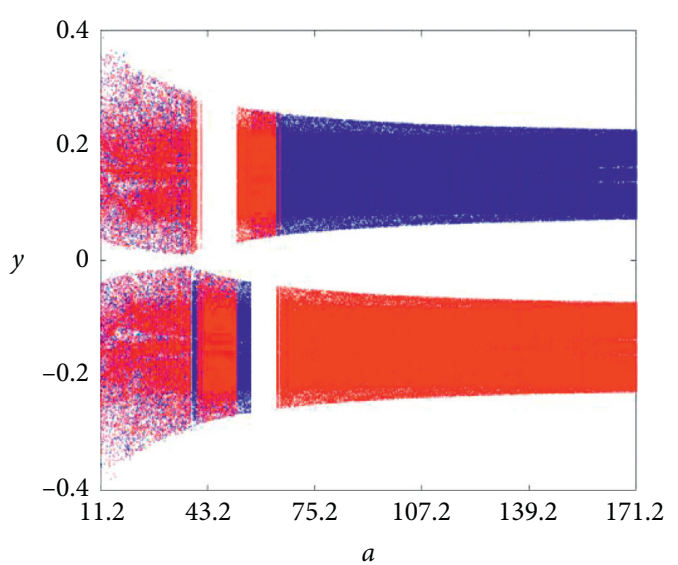

(a)

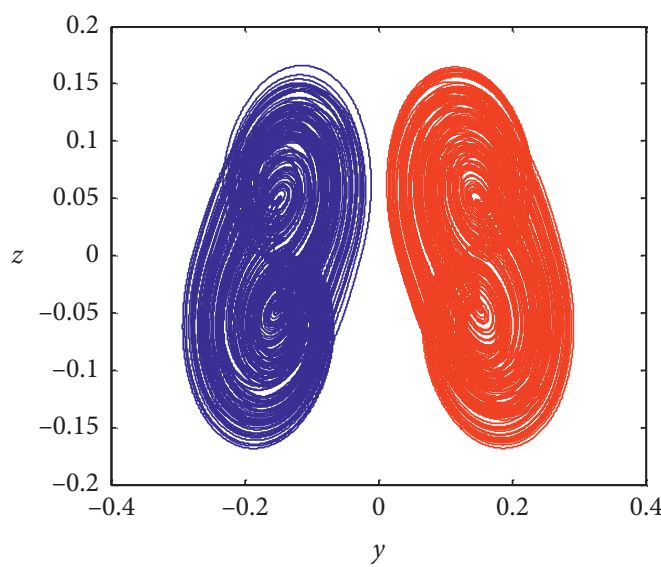

(c)

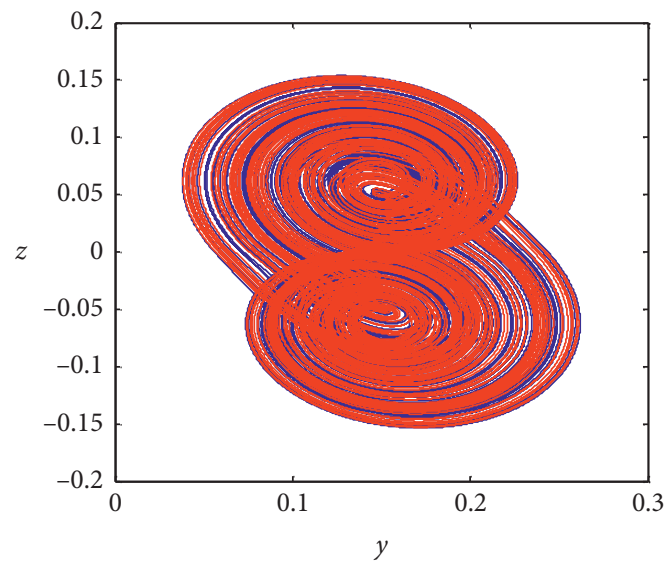

(e)

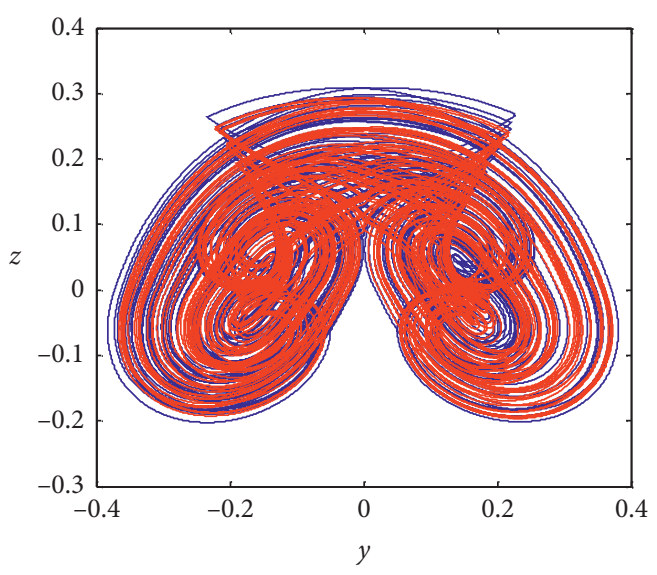

(b)

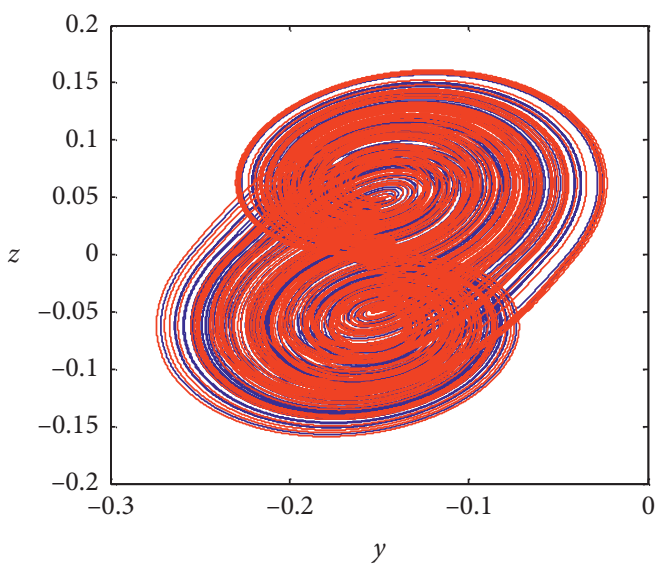

(d)

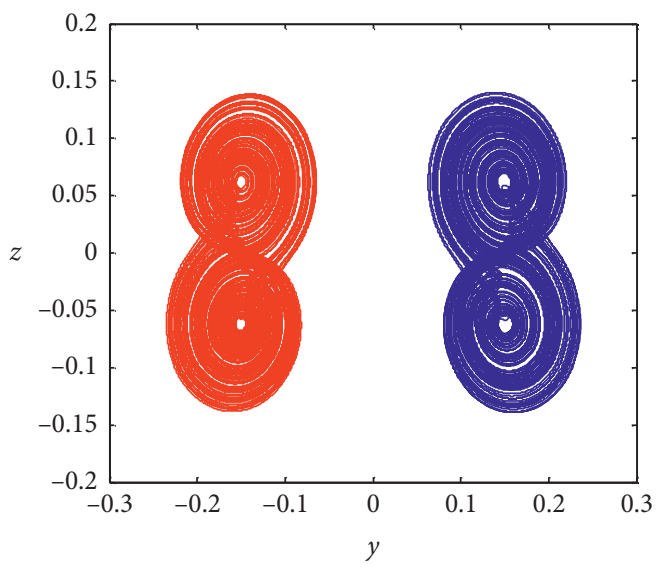

(f)

FIGURE 4: Numerical simulations for the proposed multiscroll chaotic system under mode 1: $\mathrm{M}(-,+)$ with variation of a under the initial condition $\left(x_{0}, y_{0}, z_{0}, w_{0}\right)=(0.01,0.01,0.01,0.01)$ (blue case) and $\left(x_{0}, y_{0}, z_{0}, w_{0}\right)=(0.01,-0.01,-0.01,0.01)$ (red case). (a) Bifurcation diagram, (b) $a=19.2$, (c) $a=40$, (d) $a=48$, (e) $a=60.8$, and (f) $a=112$.

and can well describe the complexity of the proposed chaotic time seriescin.

3.3. Dynamic Map of the New Chaotic System. Under $a=112$ and initial condition being $\left(x_{0}, y_{0}, z_{0}, w_{0}\right)=(0.01,0.01,0.01$, 0.01 ), dynamic map that is used to describe the dynamics of the proposed chaotic system with respect to the common influence of the varying parameters $b$ and $c$ is described in Figure 8. Obviously, there are three different color regions suggesting three different dynamic behaviors in Figure 8. The cyan area marked with $L$ indicates that when the values of parameters $b$ and $c$ are within this region, the proposed chaotic system will be in a chaotic state, and the maximum 


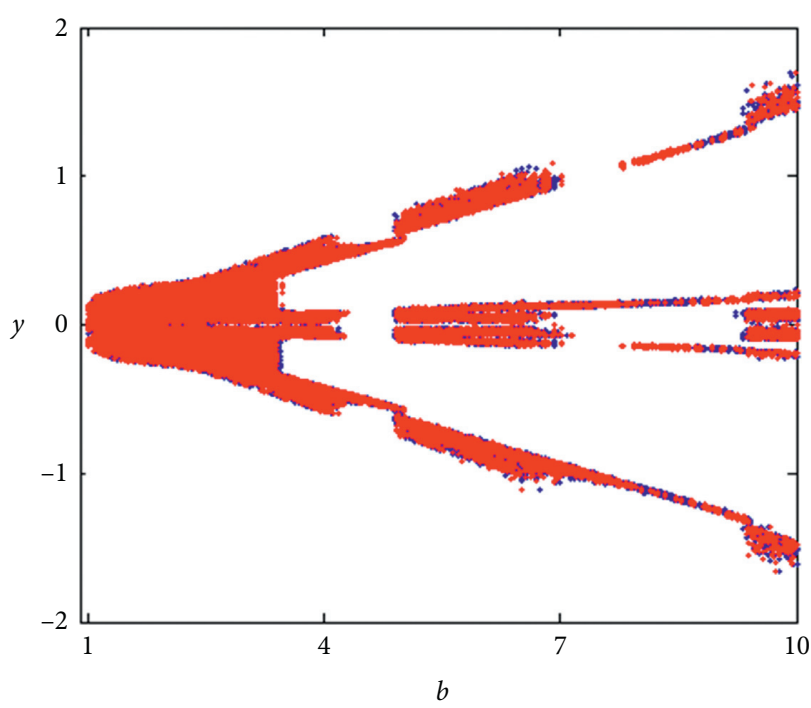

(a)

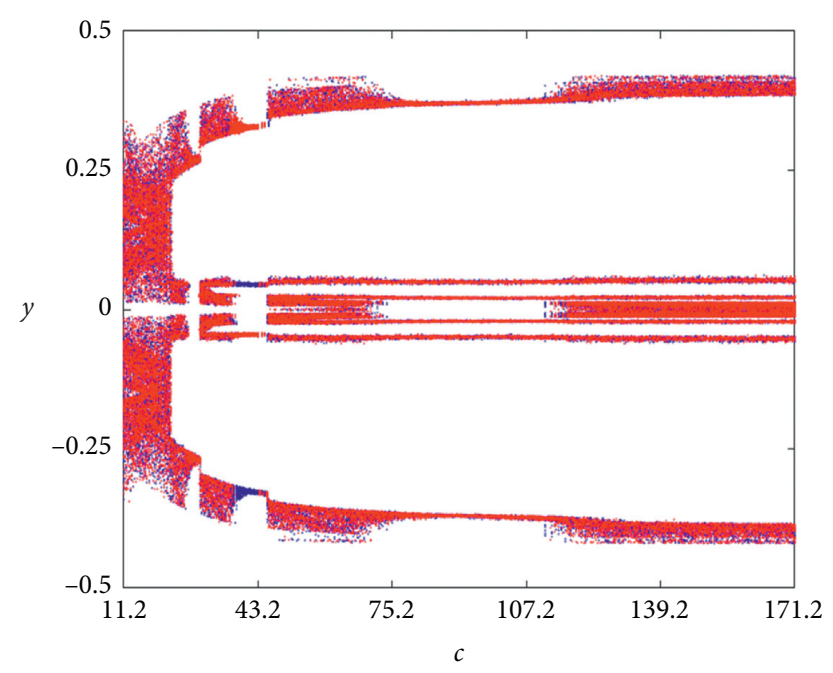

(b)

FIgURe 5: Bifurcation diagram of the proposed multiscroll chaotic system under mode $1: \mathrm{M}(-,+)$ with the initial condition $\left(x_{0}, y_{0}, z_{0}, w_{0}\right)=$ $(0.01,0.01,0.01,0.01)$ (blue case) and $\left(x_{0}, y_{0}, z_{0}, w_{0}\right)=(0.01,-0.01,-0.01,0.01)$ (red case). (a) Variation of $\mathrm{b}$ under $a=32$ and $c=16$; (b) variation of $c$ under $b=2.4$ and $c=16$.

Lyapunov exponent corresponding to this area is positive. The blue area marked with $H$ indicates that when the values of parameters $b$ and $c$ are within this region, the proposed chaotic system will be in a quasi-periodic state, and there are two maximum Lyapunov values, both of which are zero corresponding to the blue area. The green area marked with $G$ indicates that the proposed chaotic system will be in a periodic state under these parameters values, while there is only one maximum Lyapunov exponent whose value is zero in the green region. Hence, the dynamics of the proposed chaotic system for various parameters $b$ and $c$ can be vividly described in the dynamics map.

Then different parameters $b$ and $c$ are selected in three regions of the dynamic map of the proposed chaotic system, and the maximum Lyapunov exponents for different parameters are calculated. The calculated results are summarized in Table 1 , where the first point $b=2.4$ and $c=16$ is chosen in the $L$ region, the second point $b=1.5$ and $c=33$ is chosen in the $H$ region, and the third point $b=3$ and $c=55$ is chosen in the $G$ region. According to the calculated results, one can judge that the proposed chaotic system moves in chaotic state, quasi-periodic state, or periodic state when the different parameters are chosen. In order to verify the maximum Lyapunov exponents of the proposed chaotic system under different parameters, we have given the timedomain diagrams and phase diagrams of this system in three different dynamical regions, as shown in Figure 7. Hence, based on the relationship [29] between the dynamical characteristics of the nonlinear system and the values of the Lyapunov exponents, one can see that the dynamic behaviors of the proposed chaotic system are consistent with the results judged by the calculated Lyapunov exponents. Besides, the analysis method described above can also be employed to investigate the dynamics of the proposed chaotic system with respect to other different parameters.
3.4. Attraction Basin and Multistability. The attraction domain is an important tool to analyze coexisting attractors with respect to different initial states of the dynamical system. Here, under $a=112, b=2.4, c=16, x_{0}=0.01$, and $w_{0}=0.01$, the attraction basin of the proposed chaotic system is described in Figure 9. One can see that the basin of attraction contains two different color regions, indicating the coexisting characteristic of different state attractors; that is, the proposed chaotic system is of multistability. Among them, the pink region marked with $E$ suggests that if the initial state starts from this region, the proposed chaotic system will be in one chaotic state. However, the yellow region marked with $F$ indicates that if the initial state starts from this region, the proposed chaotic system will be in another chaotic state, which is symmetric to chaotic state in region $E$. That is to say, the initial trajectories of the proposed chaotic system from the pink region or the yellow region will enter two the symmetric chaotic motion states eventually and respectively.

As shown in Case 5 of Section 3.1, where the initial condition $y_{0}=0.01$ and $z_{0}=0.01$ is chosen in region $F$, the initial condition $y_{0}=-0.01$ and $z_{0}=-0.01$ is chosen in region $E$, respectively; they finally display two symmetric coexisting chaotic attractors in Figure 4(f). Hence, there is multistability in the proposed chaotic system whose motion trajectories starting from different initial states in chaotic region (pink and yellow regions) always enter one of the symmetric chaotic states.

\section{Multiscroll Chaotic Attractors Generation}

In this section, the system parameters are designed to generate multiscroll chaotic attractors. From the theoretical analysis of four-scroll chaotic attractors generation in system (2) under $n=0$, one can see that system (2) has 6 equilibrium points 


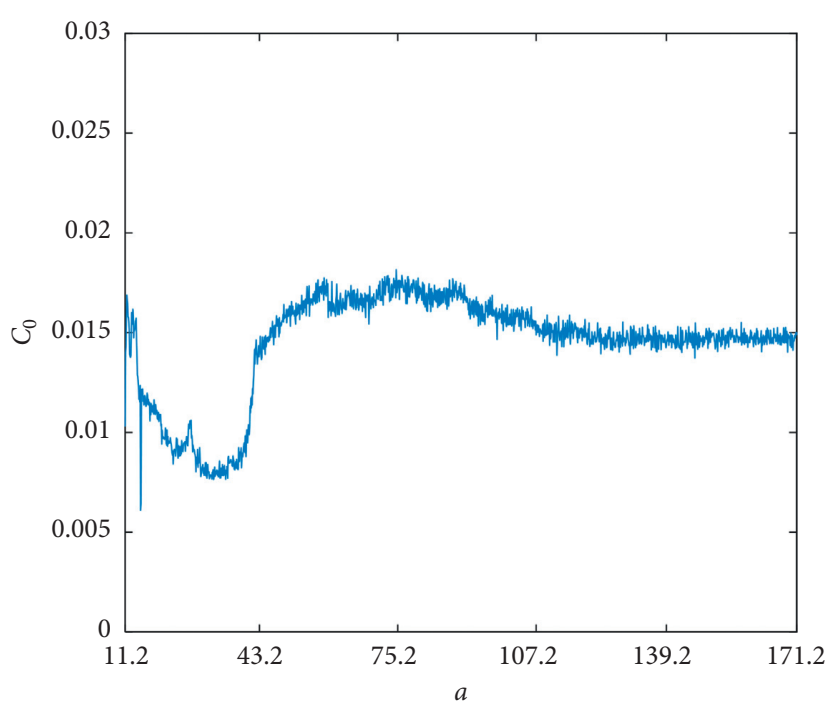

(a)

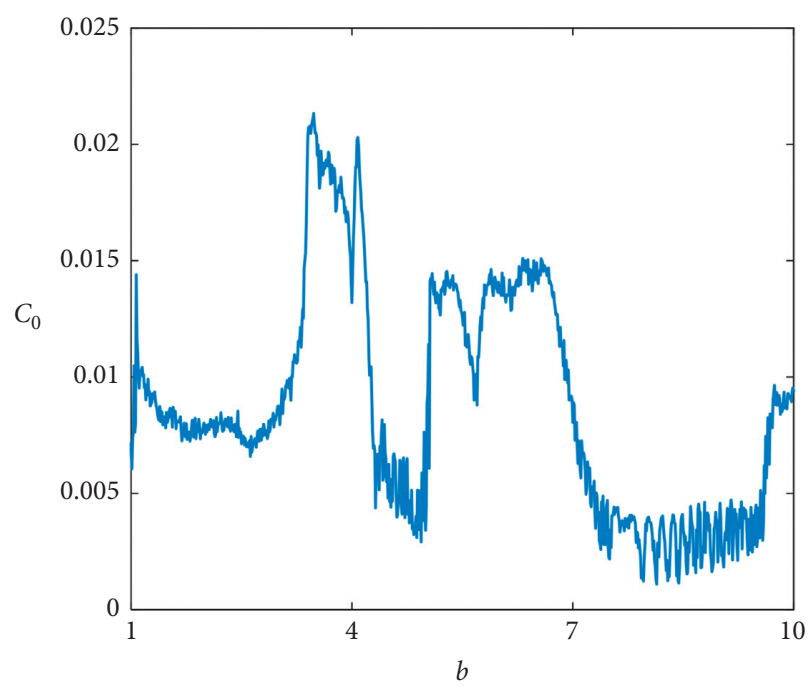

(b)

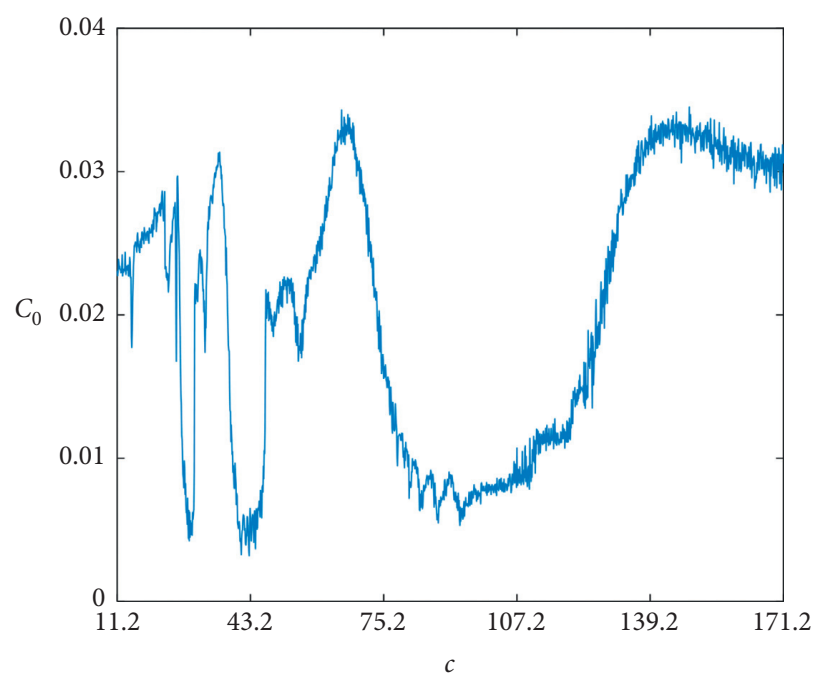

(c)

Figure 6: $C_{0}$ complexity analysis of the proposed chaotic system under mode $1: M(-,+)$ for the initial condition being $\left(x_{0}, y_{0}, z_{0}, w_{0}\right)=(0.01$, $0.01,0.01,0.01$ ). (a) Related to parameter a when $b=2.4$ and $c=16$. (b) Related to parameter $\mathrm{b}$ when $a=32$ and $c=16$. (c) Related to parameter $\mathrm{c}$ when $a=32$ and $b=2.4$.

TABLE 1: Lyapunov exponents of the proposed chaotic system with varying parameters under $a=11$.

\begin{tabular}{|c|c|c|c|c|c|c|}
\hline \multirow{2}{*}{ Parameter values } & \multicolumn{4}{|c|}{ Lyapunov exponents } & \multirow{2}{*}{ Dynamics } & \multirow{2}{*}{ Curves } \\
\hline & $L E_{1}$ & $L E_{2}$ & $L E_{3}$ & $L E_{4}$ & & \\
\hline$b=2.4, c=16$ & 1.33 & 0 & -15.06 & -114.07 & Chaos & Figures $7(a)$ and $7(b)$ \\
\hline$b=1.5, c=33$ & 0 & 0 & -30.49 & -114.16 & Quasi-period & Figures $7(\mathrm{c})$ and $7(\mathrm{~d})$ \\
\hline$b=3, c=55$ & 0 & -1.71 & -54.66 & -108.59 & Period & Figures $7(\mathrm{e})$ and $7(\mathrm{f})$ \\
\hline
\end{tabular}

where two of them are unstable saddle point and the remaining four are index- 2 saddle foci. For the unstable point, it cannot form the scroll attractors, since the trajectory of the system will be away from it quickly. So, the four index-2 saddle foci are essential to generate the four-scroll chaotic attractors. Thus, in order to obtain the multiscroll chaotic attractors, it is necessary 

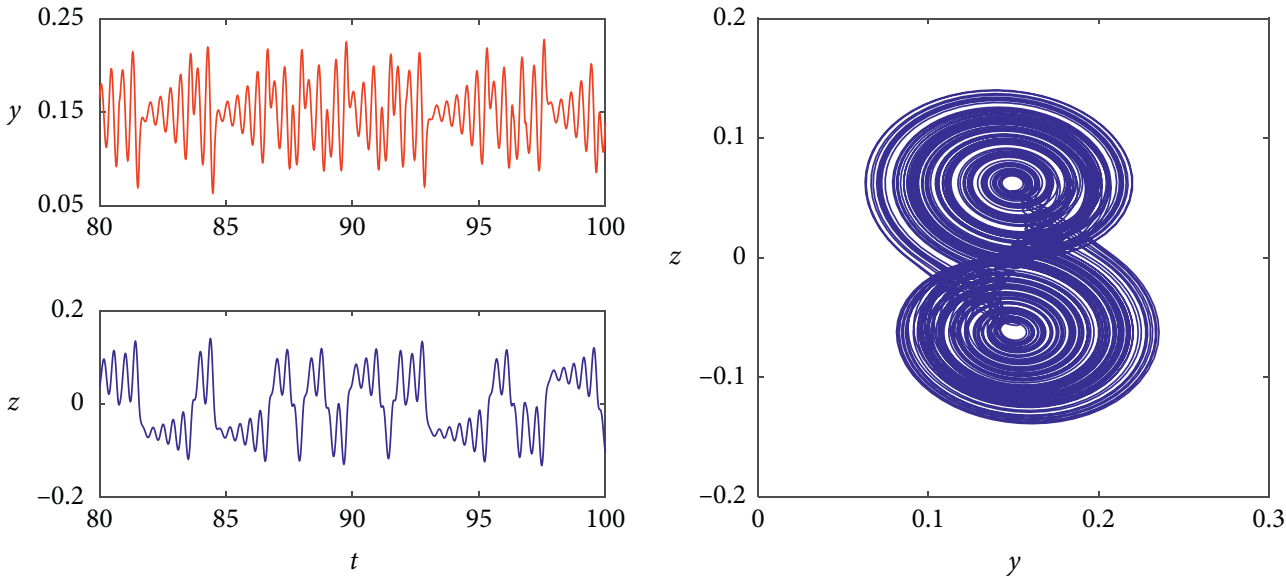

(a)

(b)
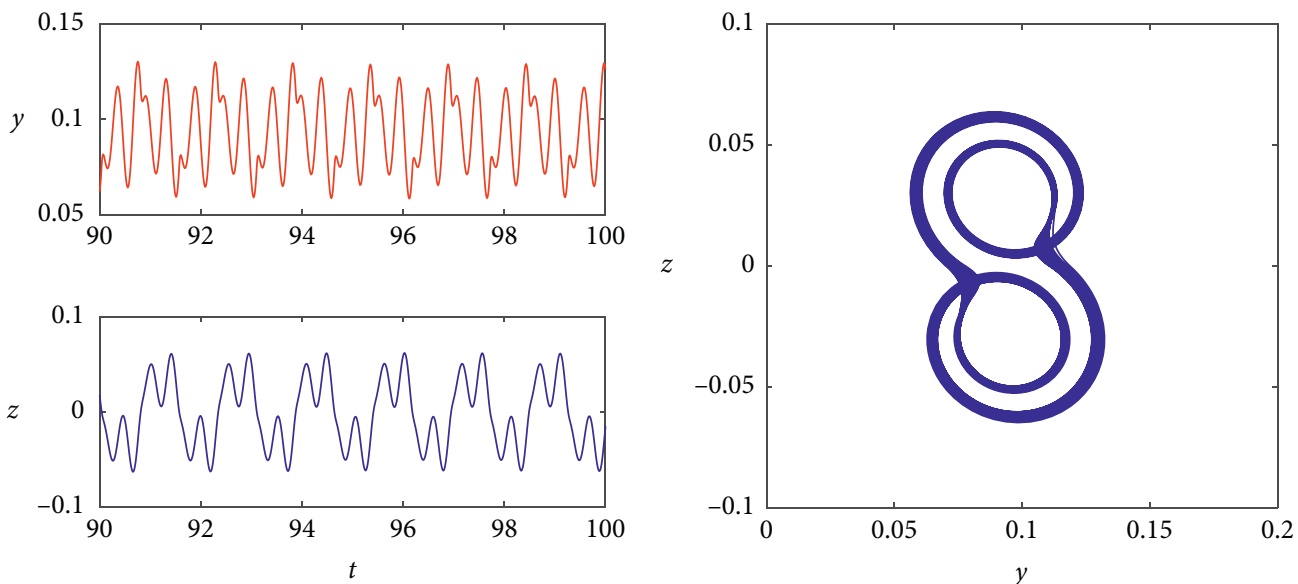

(c)

(d)
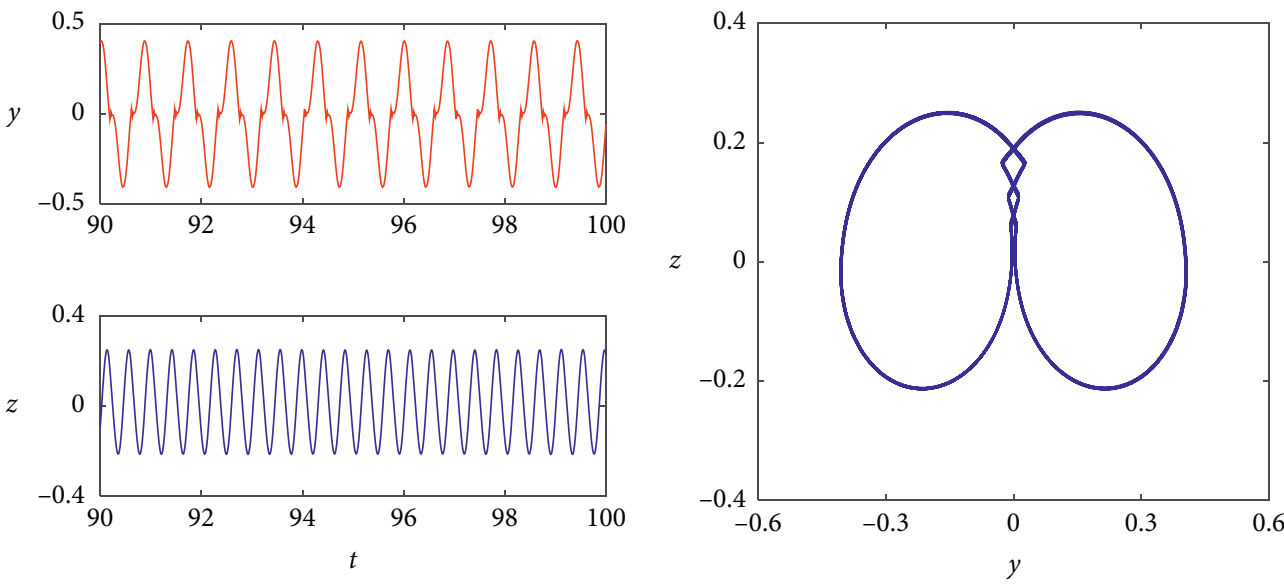

(e)

(f)

FIGURE 7: Different dynamics of the proposed chaotic system with respect to the initial condition being $\left(x_{0}, y_{0}, z_{0}, w_{0}\right)=(0.01,0.01,0.01,0.01)$ and $a=112$. For $b=2.4$ and $\mathrm{c}=16$, (a) time-domain waveforms and (b) chaotic attractor. For $b=1.5$ and $c=33$, (c) time-domain waveforms and (d) quasi-periodic attractor. For $b=3$ and $c=55$, (e) time-domain waveforms and (f) periodic attractor.

to add the index-2 saddle foci to the system. Here, let $a=32$, $b=2.4$, and $c=16$, and changing the values of $n$, the $4 n+4$ scroll chaotic attractors can be generated. For example, let $n=1$; the equilibrium points of system (2) are 


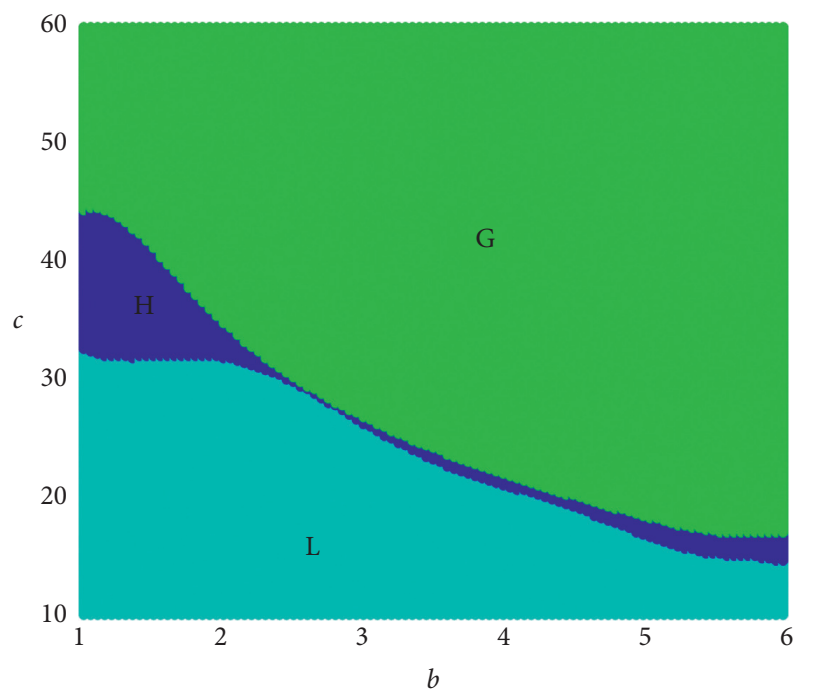

Figure 8: Dynamic map of the proposed chaotic system under mode $1: M(-,+)$ under $a=112$.

$$
[X, Y, Z, W]=\left\{\begin{array}{l}
S_{+3,+4}=(( \pm b / 16),( \pm b / 16),(3 / c),(3 / c)), \quad \text { with } z>0.125 \\
S_{+01,+02}=(( \pm b / 16),( \pm b / 16),(2 / c),(2 / c)), \quad \text { with } z=0.125 \\
S_{+1,+2}=(( \pm b / 16),( \pm b / 16),(1 / c),(1 / c)), \quad \text { with } 0<z<0.125 \\
S_{+0,-0}=(( \pm b / 16),( \pm b / 16), 0,0), \quad \text { with } z=0, \\
S_{-1,-2}=(( \pm b / 16),( \pm b / 16),(-1 / c),(-1 / c)), \quad \text { with }-0.125<z<0 \\
S_{-01,-02}=(( \pm b / 16),( \pm b / 16),(-2 / c),(-2 / c)), \quad \text { with } z=-0.125 \\
S_{-3,-4}=(( \pm b / 16),( \pm b / 16),(-3 / c),(-3 / c)), \quad \text { with } z<-0.125
\end{array}\right.
$$

The Jacobian matrix of system (2) at the equilibrium points is

$$
J=\left[\begin{array}{ccccc}
-a & a & 0 & 0 \\
\mp 128 \delta(4 X) Z & \pm 128 \delta(4 Y) W & \mp 16 \operatorname{sgn}(4 X) & \pm 16 \operatorname{sgn}(4 Y) & \\
\pm 16 \operatorname{sgn}(X) & 0 & 0 & 0 & \delta(4 Z+0.5 k)-c \\
0 & 0 & 8 \sum_{k=-1}^{1} &
\end{array}\right]
$$

From equation (10) and the definition of $\delta()$, one can see that it has $\delta(4 Z-0.5)$ whose value will be infinite under $Z=0.125, \delta(4 Z)$ whose value will be infinite under $Z=0$, and $\delta(4 Z+0.5)$ whose value will be infinite under $Z=-0.125$. Thus, under these three cases, the corresponding eigenvalues of equation (10) can also not be calculated.

Of course, the same technique in Section 2 can be used and the same results can be obtained; that is, $S_{+01,+02}, S_{+0 \text {,- }}$ 0 , and $S_{-01,-02}$ are unstable point. Hence, the system's trajectory will be departed rapidly at these six equilibrium points. However, for $S_{+3,+4}, S_{+1,+2}, S_{-1,-2}$, and $S_{-3,-4}$, the corresponding eigenvalues of equation (10) can be obtained and the results are the same as those in Section 2. Therefore, all these eight equilibrium points are index-2 saddle foci, which are necessary to form eight-scroll chaotic attractors. For example, based on $a=32, b=2.4, c=16$, and $n=1$ and under the initial condition $\left(x_{0}, y_{0}, z_{0}, w_{0}\right)=(0.01,0.01,0.01$, 0.01 ), the numerical simulations by using the ode 45 algorithm with variable step size and the simulation time $T=1000$ s with time step being 0.002 s in Matlab software are shown in Figure 10. One can see that the proposed multiscroll chaotic system has eight-scroll chaotic attractors. In addition, for the same conditions of simulation algorithm and simulation time, the calculated Lyapunov exponents of the proposed multiscroll chaotic system under this case are $L E_{1}=2.76, L E_{2}=0, L E_{3}=-11.37$, and $L E_{4}=-39.35$. 


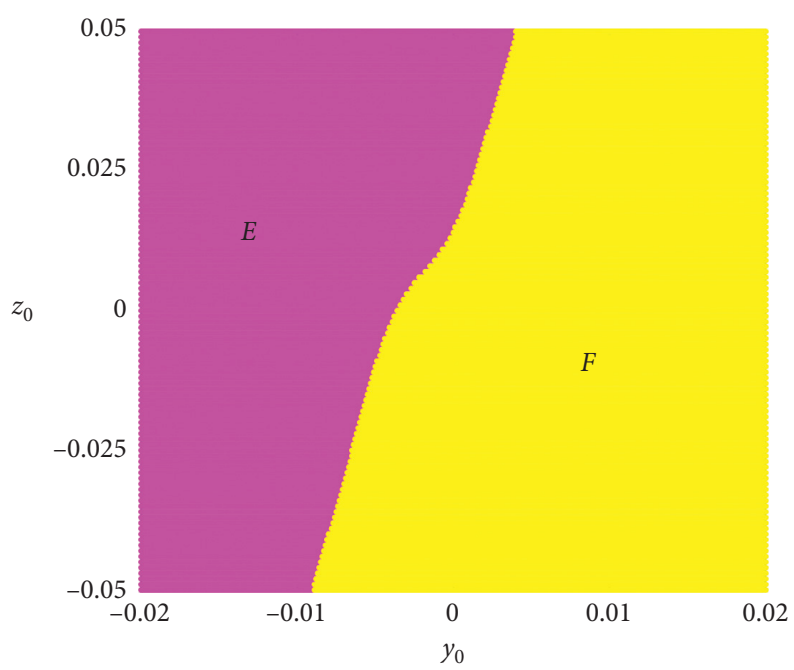

Figure 9: The basin of attraction of the proposed chaotic system under mode $1: M(-,+)$ under $a=112, b=2.4, c=16, x_{0}=0.01$, and $w_{0}=0.01$.

\section{FPGA Implementation}

In this section, the proposed multiscroll chaotic system is implemented by using FPGA. Here, the second-order Runge-Kutta method and the fixed-point data format $32 \mathrm{Q} 26$, where 1 bit is the sign part, 5 bits is the integer part, and 26 bits is the decimal part, are applied to calculate the multiscroll chaotic system.

5.1. Two-Stage Iteration. Based on the second-order Runge-Kutta method, the discretization about system (2) under $\mathrm{M}(-,+)$ can be derived for implementing it in FPGA and its results are

$$
\left\{\begin{array}{l}
x_{k+1}=x_{k}+\Delta t \times\left(a\left(y_{k}-x_{k}\right)\right), \\
y_{k+1}=y_{k}+\Delta t \times\left(-16 z_{k} \operatorname{sgn}\left(4 x_{k}\right)+16 w_{k} \operatorname{sgn}\left(4 y_{k}\right)\right), \\
z_{k+1}=z_{k}+\Delta t \times\left(16\left|x_{k}\right|-b\right), \\
w_{k+1}=w_{k}+\Delta t \times\left(-c w_{k}+\sum_{k=-n}^{n} \operatorname{sgn}\left(4 z_{k}+0.5 k\right)\right),
\end{array}\right.
$$

where $\Delta t$ is the discrete time step. Defining $V_{k}=\left(x_{k}, y_{k}, z_{k}, w_{k}\right)$ ${ }^{T}$, the proposed multiscroll chaotic system can be expressed as follows:

$$
V_{k+1}=V_{k}+\Delta t \times G\left(V_{k}\right) .
$$

Based on the second-order Runge-Kutta method [30], there are two stage iteration routines. In stage 1 , the state vector is updated as follows:

$$
V_{k+(1 / 2)}=V_{k}+\frac{h}{2} \times G\left(V_{k}\right),
$$

where $h$ is the discrete time gap and $h=1 / 128$ is taken for the implementation in experimental results. $V_{k+1 / 2}$ indicates the intermediate results in a half step, that is, $\Delta t=h / 2$.
In stage 2, $V_{k+1}$ is updated based on $V_{k+1 / 2}$ from stage 1 , and its formula is as follows:

$$
V_{k+1}=V_{k}+h \times G\left(V_{k+(1 / 2)}\right)
$$

5.2. Verilog HDL Implementation. Here, Verilog HDL language $[31,32]$ is used to realize the digital chaotic system in FPGA. Notably, in programming, after initial state is defined, state machine method is applied to split the whole digital chaotic generator into several subprocesses, which includes nine states $\left(\mathrm{S}_{0}, \mathrm{~S}_{1}, \mathrm{~S}_{2}, \mathrm{~S}_{3}, \mathrm{~S}_{4}, \mathrm{~S}_{5}, \mathrm{~S}_{6}, \mathrm{~S}_{7}\right.$, and $\left.\mathrm{S}_{8}\right)$ and the corresponding state machine flowchart is shown in Figure 11. We have the following:

(i) $\mathrm{S}_{0}$ : calculation about $4 x, 4 y$, and $4 z$ by using shift operation.

(ii) $\mathrm{S}_{1}$ : implementation of sgn $(4 x)$, sgn $(4 y),|x|$, and sgn $(4 z+0.5 k)$ operations where $k=-n: n$.

(iii) $\mathrm{S}_{2}$ : add up all sgn $(4 z+0.5 k)$, parallel implementation of $a x$ and ay operations, and parallel implementations to get the results of 16sgn $(4 x) z$ and 16sgn (4y)w.

(iv) $S_{3}$ : parallel implementations to get the results of $-c w$ and $f(z)$.

(v) $S_{4}$ : parallel implementations to get the results of $\dot{x}$, $\dot{y}, \dot{z}$, and $\dot{\mathrm{w}}$.

(vi) $S_{5}$ : select 32 bits to present $\dot{x}, \dot{y}, \dot{z}$, and $\dot{w}$.

(vii) $S_{6}$ : according to the second-order Runge-Kutta method expressed in equations (13) and (14), the variables $\dot{x}, \dot{y}, \dot{z}$, and $\dot{w}$ are iterated. Obviously, there are two iterative stages. When the iteration is in stage 1 , implement the operation in equation (13) and then direct to state $S_{0}$. When the iteration is in stage 2 , implement the operation in equation (14) and then direct to state $S_{7}$. 


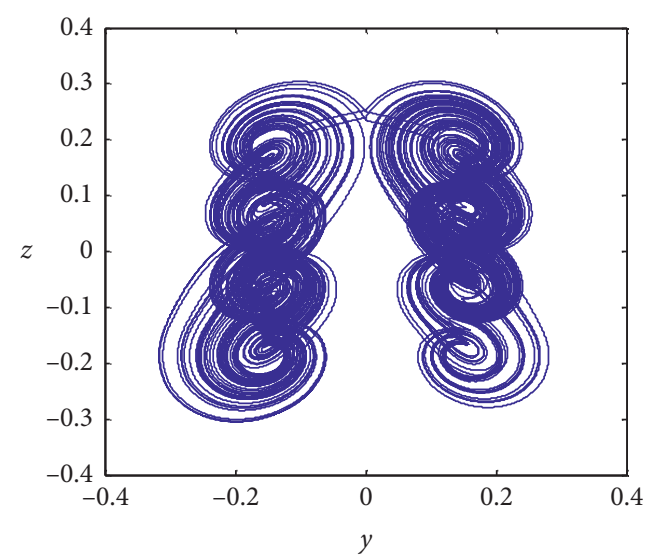

(a)

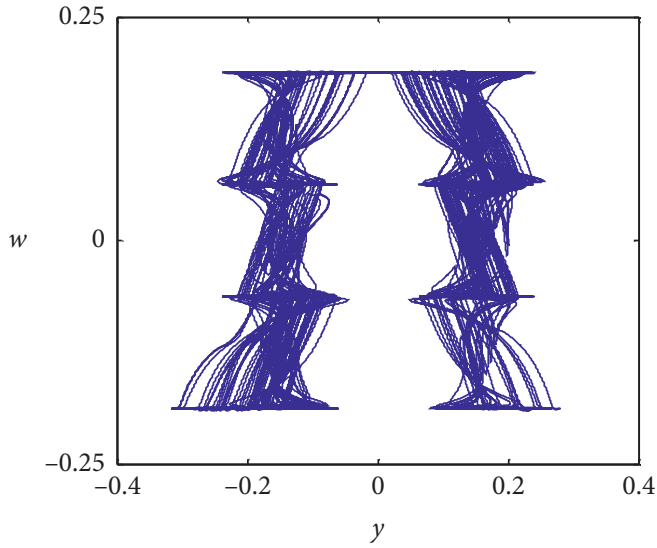

(b)

Figure 10: Numerical simulations for the chaotic system $n=1$. (a) $y$ versus $z$; (b) $y$ versus $w$.

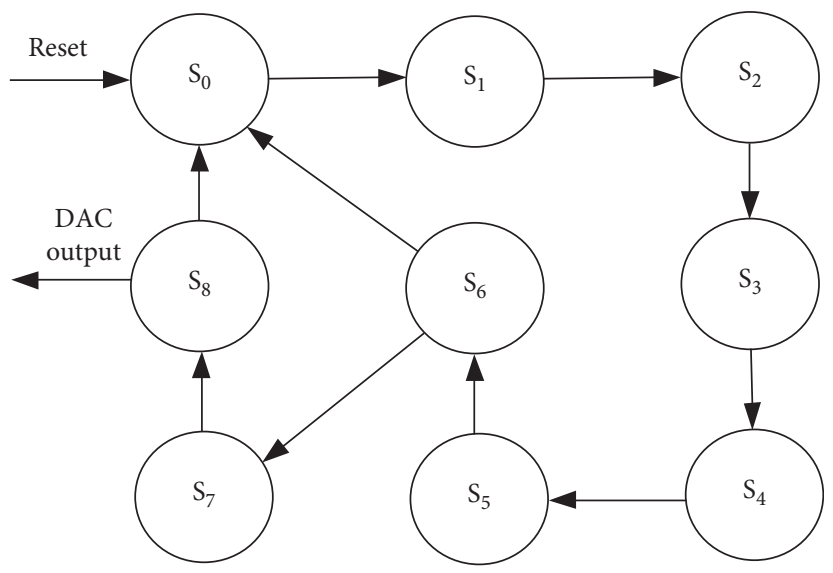

FIGURE 11: State machine flowchart.

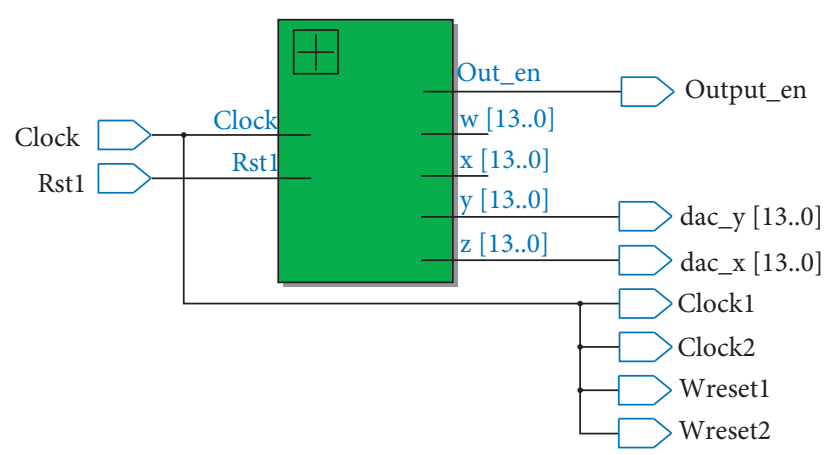

Figure 12: Top-layer RTL of the proposed chaotic system.

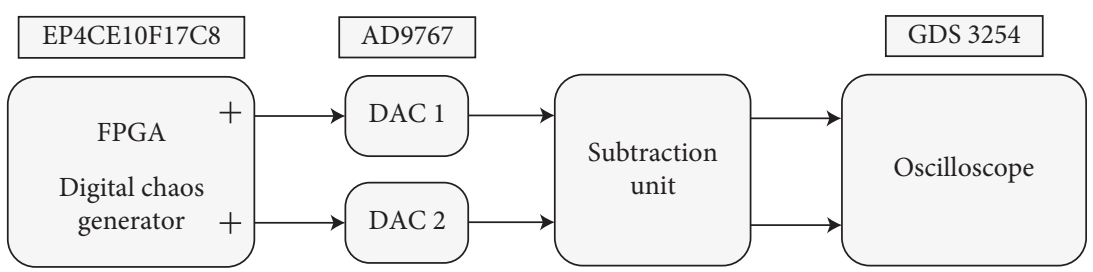

FIGURE 13: The architecture of experimental hardware platform for the proposed chaotic system. 


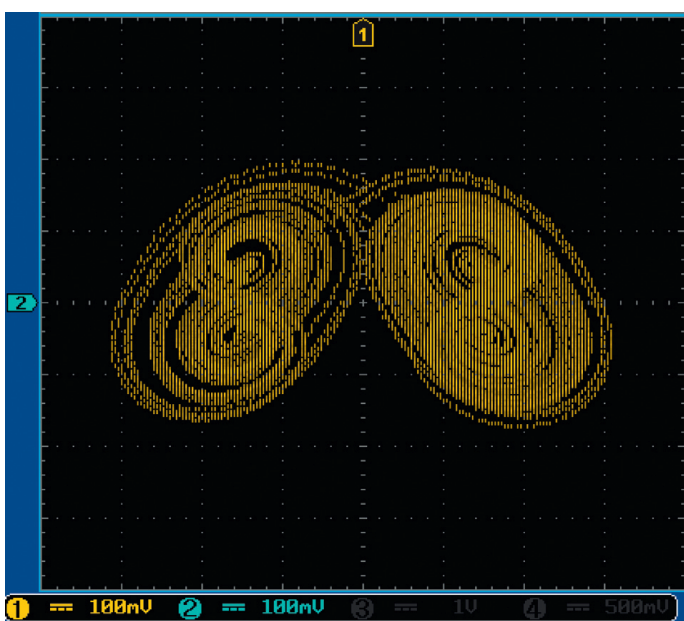

(a)

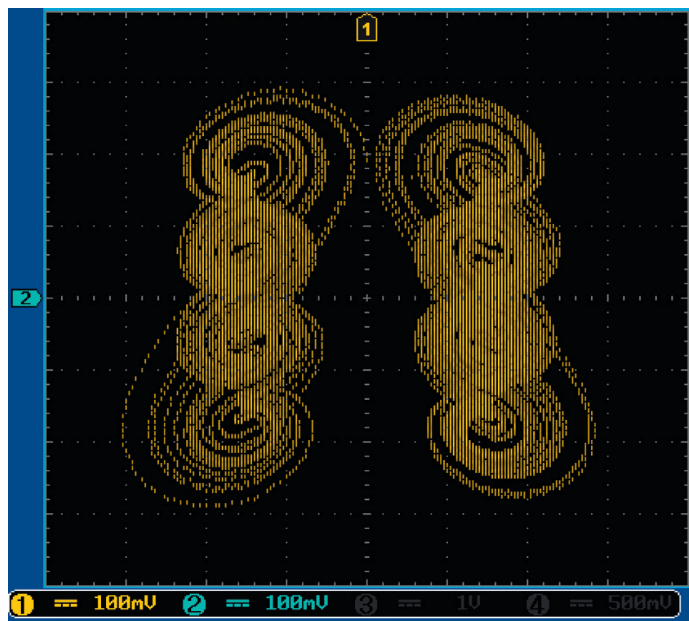

(c)

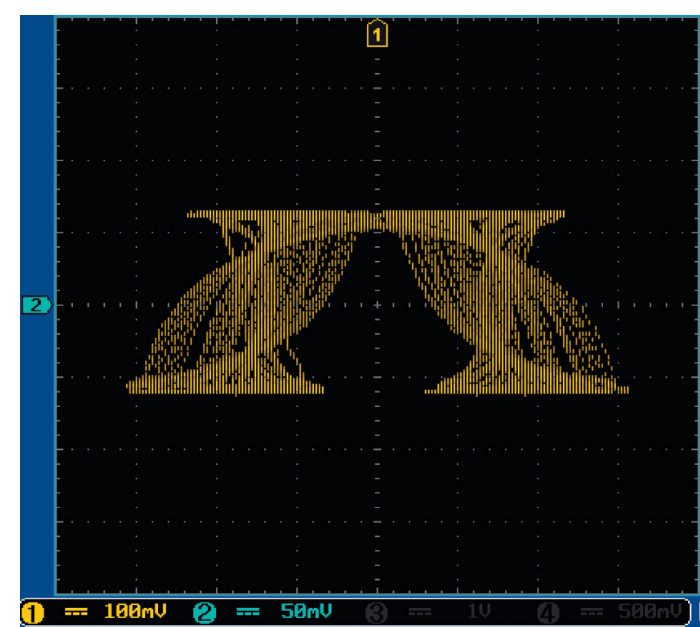

(b)

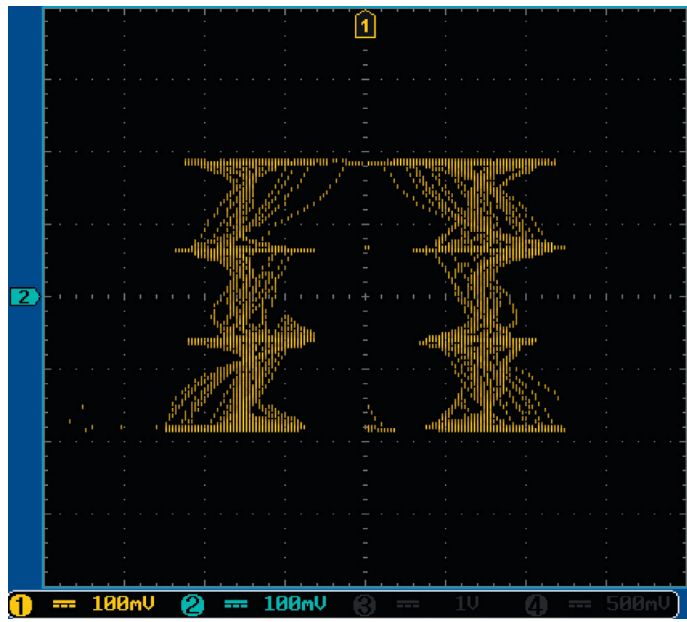

(d)

FIGURE 14: Phase portraits of chaotic system with $n=0$ and $n=1$ from the experimental results: (a) $y$ versus $z$ with $n=0$, (b) $y$ versus $w$ with $n=0$, (c) $y$ versus $z$ with $n=1$, and (d) $y$ versus $w$ with $n=1$.

(viii) $\mathrm{S}_{7}$ : add a positive offset to the results obtained in state $S_{6}$ to avoid negative outputs.

(ix) $\mathrm{S}_{8}$ : output the digital chaotic signal to the external digital-to-analog converter (DAC) chip and direct to state $\mathrm{S}_{0}$.

The top-layer RTL viewer of the realization is shown in Figure 12, where we have the following: "clock" (the clock for FPGA, the input clock is set as $50 \mathrm{MHz}$ in our experiments), "rst1" (the reset for FPGA), "clock1" and "clock2" (the clock for external DAC chip), "wreset1" and "wreset2" (the reset for external DAC chip), "ouput_en" (the output is valid under the high level signal), "x[13..0]" (the digital output of $x$ in each step), "y[13..0]" (the digital output of $y$ in each step), "z[13..0]" (the digital output of $z$ in each step), "w [13..0]" (the digital output of $w$ in each step), "dac_y[13..0]" (the output of y[13..0] to the external DAC chip), and " $\mathrm{dac} \_\mathrm{x}$ [13..0]" (the output of $z[13 . .0]$ to the external DAC chip).

5.3. Experimental Results. After writing the Verilog HDL program for the multiscroll chaotic system successfully, this program can be downloaded into FPGA via USB Blaster so that it is converted to a hardware list and then be configured into FPGA to form the corresponding digital circuits. The Altera Cyclone IV EP4CE10F17C8 FPGA, which has available internal resources including 10320 logic elements, 423936 RAMs implement bits, 2 PLLs, and 46 hardware multipliers, is adopted. Also the external DAC chip AD9767 (14 bits) with two output channels is applied in the hardware platform.

The architecture of experimental hardware platform for the proposed chaotic system is shown in Figure 13. Notably, the proposed chaotic system can generate chaotic attractors containing negative signals; however, the digital chip AD9767 whose conversion rate up to $125 \mathrm{Msps}$ can only process positive signals, so that a positive number should be added for each output of FPGA (+ in Figure 13). In order to keep consistency with the simulation results, the DAC output signal needs to be subtracted by the same positive number; that is, a subtraction unit before being captured by the digital oscilloscope GDS 3254 should be needed. The experimental results based on FPGA technology are shown 
TABLE 2: Hardware resources required to implement the proposed multiscroll chaotic system.

\begin{tabular}{lr}
\hline Terms & Resource consumptions \\
\hline Logic elements & $2303 / 10320(22 \%)$ \\
Registers & $1816 / 10320(18 \%)$ \\
RAMs implement bits & $100352 / 423936(24 \%)$ \\
Multipliers elements & $0 / 46(0 \%)$ \\
PLLs & $0 / 2(0 \%)$ \\
Throughput (Mbits/s) & 100 \\
Clock cycles by iteration & 16 \\
Latency (ns) & 320 \\
\hline
\end{tabular}

TABLE 3: Summary of the required resources to implement different chaotic systems by using FPGA.

\begin{tabular}{lccccc}
\hline Ref. & Iterative approach & Logic elements & Registers & Multiplier elements & Max. freq. (MHz) \\
\hline$[33]$ & Runge-Kutta 4 & 4422 & 1891 & 24 & 69.50 \\
{$[34]$} & Runge-Kutta 4 & 4708 & 2662 & 208 & 84.77 \\
This work & Runge-Kutta 2 & 2303 & 1816 & 0 & 75.59 \\
\hline
\end{tabular}

in Figure 14. By comparing Figures 14(a), 14(b), 14(c), and 14(d) with Figures 1(a), 1(b), 10(a), and 10(b), respectively, one can see that the results from these two sides are in good agreement with each other.

In our experiments, the input clock is set as $50 \mathrm{MHz}$ and the total thermal power dissipation is obtained as $77.01 \mathrm{~mW}$ by employing the PowerPlay Power Analyzer Tool in the software Quartus II. The other hardware resources required to implement the new multiscroll chaotic system based on FPGA chip are listed in Table 2. In this FPGA implementation, $22 \%$ of the logic elements, $18 \%$ of the logic registers, $24 \%$ of the RAMs, $0 \%$ of the embedded multipliers, and $0 \%$ of the PLLs of the Altera Cyclone IV EP4CE10F17C8 FPGA are occupied, where the resource utilization rates are determined by the ratio of the required number of resources to the total number of resources in the Altera Cyclone IV EP4CE10F17C8 FPGA board. Besides, the throughput of this digital implementation is given as $100 \mathrm{Mbits} / \mathrm{s}$, the last two lines provide that there are 16 clock cycles required to evaluate a single iteration, and the delay time is given as 320 nanoseconds when using a $50 \mathrm{MHz}$ clock signal. In addition, the comparisons on FPGA implementations between some existent chaotic systems given in $[33,34]$ and the proposed chaotic system are shown in Table 3. In summary, one can see that the calculation accuracy of the second-order Runge-Kutta method is sufficient to meet the requirements of the proposed chaotic system, and it requires fewer FPGA's resources. Hence, in this paper, the FPGA realization is feasible, the resource consumption is reasonable, the operation is stable and the output speed is fast.

\section{Conclusion}

Based on the modified Lorenz-type system and adding an additional variable, a novel chaotic system that can generate multiscroll chaotic attractors is proposed. Attraction basin, bifurcation diagram, dynamic map, phase portrait, Lyapunov exponent spectrum, and $C_{0}$ complexity are carried out for this proposed chaotic system to analyze its dynamical behaviors. Research results show that there are some typical dynamics including multistability, bifurcation, chaotic attractors, quasi-periodic attractor, and periodic attractor in this proposed chaotic system. For the different initial conditions, symmetric coexisting attractors will occur in this system. Moreover, theoretical analysis, numerical simulations, and FPGA implementations together demonstrate that the $4 n+4$ scroll chaotic attractors can be obtained by selecting the value of the natural number $n$. Compared with other existent FPGA implementations of the chaotic generators, the proposed multiscroll chaotic system has an advantage of requiring less resources, especially only requiring $0 \%$ of the embedded multipliers and $0 \%$ of the PLLs of the FPGA chip. Hence, this work will benefit chaotic secure communications, which will be our future work.

\section{Data Availability}

The data used to support the findings of this study are available from the corresponding author upon request.

\section{Conflicts of Interest}

The authors declare that they have no conflicts of interest.

\section{Acknowledgments}

This work was supported in part by the New Star of Youth Science and Technology of Shaanxi Province under Grant 2016KJXX-40.

\section{References}

[1] W. K. S. Tang, G. Q. Zhong, G. Chen, and K. F. Man, "Generation of $\mathrm{N}$-scroll attractors via sine function," IEEE Transactions on Circuits and Systems I: Fundamental Theory and Applications, vol. 48, no. 11, pp. 1369-1372, 2001.

[2] E. Günay and R. Kiliç, "A new way of generating $N$-scroll attractors via trigonometric function," International Journal of Bifurcation and Chaos, vol. 21, no. 03, pp. 897-901, 2011. 
[3] S. Özoğuz, A. S. Elwakil, and K. N. Salama, "N-scroll chaos generator using nonlinear transconductor," Electronics Letters, vol. 38, no. 14, pp. 685-686, 2002.

[4] J. Lü and G. Chen, "Generating multiscroll chaotic attractors: theories, methods and applications," International Journal of Bifurcation and Chaos, vol. 16, no. 04, pp. 775-858, 2006.

[5] Y. Huang, Y. Chen, K. Li et al., "Multi scrolls chaotic encryption for physical layer security in OFDM-PON," Optics Communications, vol. 471, p. 126009, 2020.

[6] A. Atangana, G. Bouallegue, and K. Bouallegue, "New multiscroll attractors obtained via Julia set mapping," Chaos, Solitons \& Fractals, vol. 134, Article ID 109722, 2020.

[7] D. Mathale, E. F. Doungmo Goufo, and M. Khumalo, "Coexistence of multi-scroll chaotic attractors for fractional systems with exponential law and non-singular kernel," Chaos, Solitons \& Fractals, vol. 139, Article ID 110021, 2020.

[8] J. H. Lü, S. M. Yu, H. Leung, and G. R. Chen, "Experimental verification of multidirectional multiscroll chaotic attractors," IEEE Transactions on Circuits and Systems I: Fundamental Theory and Applications, vol. 53, no. 1, pp. 149-165, 2006.

[9] X. Liu, X. S. Shen, and H. Zhang, "Multi-scroll chaotic and hyperchaotic attractors generated from Chen system," International Journal of Bifurcation and Chaos, vol. 22, no. 02, Article ID 1250033, 2012.

[10] C. H. Wang, H. Xu, and F. Yu, "A novel approach for constructing high-order chua's circuit with multi-directional multi-scroll chaotic attractors," International Journal of Bifurcation and Chaos, vol. 23, no. 3, Article ID 1350022, 2013.

[11] Q. Lai, Z.-H. Guan, Y. Wu, F. Liu, and D.-X. Zhang, "Generation of multi-wing chaotic attractors from a Lorenz-like system," International Journal of Bifurcation and Chaos, vol. 23, no. 09, Article ID 1350152, 2013.

[12] X. Luo, C. Wang, and Z. Wan, "Grid multi-wing butterfly chaotic attractors generated from a new 3-D quadratic autonomous system," Nonlinear Analysis: Modelling and Control, vol. 19, no. 2, pp. 272-285, 2014.

[13] N. Yu, Y.-W. Wang, X.-K. Liu, and J.-W. Xiao, "3D grid multiwing chaotic attractors," International Journal of Bifurcation and Chaos, vol. 28, no. 04, Article ID 1850045, 2018.

[14] F. R. Tahir, R. S. Ali, V.-T. Pham, A. Buscarino, M. Frasca, and L. Fortuna, "A novel 4D autonomous $2 \$ \$ \mid$ varvec $\{n\} \$ \$ n$ -butterfly wing chaotic attractor," Nonlinear Dynamics, vol. 85, no. 4, pp. 2665-2671, 2016.

[15] C. Zhang and S. Yu, "Generation of multi-wing chaotic attractor in fractional order system," Chaos, Solitons \& Fractals, vol. 44, no. 10, pp. 845-850, 2011.

[16] S. M. Yu, J. H. Lü, W. K. S. Tang, and G. R. Chen, “A general multiscroll Lorenz system family and its realization via digital signal processors," Chaos, vol. 16, no. 3, Article ID 033126, 2006.

[17] H. Cao and F. Wang, "Transient and steady coexisting attractors in a new memristor-based 4-D chaotic circuit," AEU-International Journal of Electronics and Communications, vol. 108, pp. 262-274, 2019.

[18] D. K. Shah, R. B. Chaurasiya, V. A. Vyawahare, K. Pichhode, and M. D. Patil, "FPGA implementation of fractional-order chaotic systems," AEU - International Journal of Electronics and Communications, vol. 78, pp. 245-257, 2017.

[19] P. Chen, S. Yu, X. Zhang et al., "ARM-embedded implementation of a video chaotic secure communication via WAN remote transmission with desirable security and frame rate," Nonlinear Dynamics, vol. 86, no. 2, pp. 725-740, 2016.

[20] Z. S. Lin, S. M. Yu, J. H. Lü, S. T. Cai, and G. R. Chen, “Design and ARM-embedded implementation of a chaotic map-based real-time secure video communication system," IEEE Transactions on Circuit and Systems for Video Technology Circuits, vol. 25, no. 7, pp. 1203-1216, 2015.

[21] Z. D. L. H. Mauricio, A. Leonardo, and V. Yolanda, "An experimental realization of a chaos-based secure communication using Arduino microcontrollers," The Scientific World Journal, vol. 2015, Article ID 123080, 10 pages, 2015.

[22] A. D. Pano-Azucena, J. de Jesus Rangel-Magdaleno, E. TleloCuautle, and A. de Jesus Quintas-Valles, "Arduino-based chaotic secure communication system using multi-directional multi-scroll chaotic oscillators," Nonlinear Dynamics, vol. 87, no. 4, pp. 2203-2217, 2017.

[23] M. Alçin, İ. Pehlivan, and İ. Koyuncu, "Hardware design and implementation of a novel ANN-based chaoticgenerator in FPGA," Optik, vol. 127, no. 13, pp. 5500-5505, 2016.

[24] A. S. Elwakil, S. Ozoguz, and M. P. Kennedy, "Creation of a complex butterfly attractor using a novel Lorenz-type system," IEEE Transactions on Circuits and Systems I: Fundamental Theory and Applications, vol. 49, no. 4, pp. 527-530, 2002.

[25] A. Wolf, J. B. Swift, H. L. Swinney, and J. A. Vastano, "Determining Lyapunov exponents from a time series," Physica D: Nonlinear Phenomena, vol. 16, no. 3, pp. 285-317, 1985.

[26] M. F. Danca, "Lyapunov exponents of a class of piecewise continuous systems of fractional order," Nonlinear Dynamics, vol. 81, no. 1-2, pp. 227-237, 2015.

[27] E. H. Shen, Z. J. Cai, and F. J. Gu, "Mathematical foundation of a new complexity measure," Applied Mathemtics and Mechanics-English Edition, vol. 26, no. 9, pp. 1188-1196, 2005.

[28] K. H. Sun, S. B. He, C. X. Zhu, and Y. He, "Analysis of chaotic complexity characteristics based on $C_{0}$ algorithm," Acta Physica Sinica, vol. 41, no. 9, pp. 0372-2112, 2013.

[29] J. P. Singh and B. K. Roy, "The nature of Lyapunov exponents is $(+,+,-,-)$. Is it a hyperchaotic system?” Chaos, Solitons \& Fractals, vol. 92, pp. 73-85, 2016.

[30] R. M. Wang, M. H. Liu, Y. Sheng, and J. C. Feng, "Implementation of a chaotic system based on runge-kutta algorithm and programmable gate array technology," Journal of Southwest China Normal University (Natural Science Edition), vol. 37, no. 1, pp. 41-46, 2012.

[31] E. Tlelo-Cuautle, A. D. Quintas-Valles, L. G. de la Fraga, and J. D. Rangel-Magdaleno, "VHDL descriptions for the FPGA implementation of PWL-function-based multi-scroll chaotic oscillators," Plos One, vol. 11, no. 12, Article ID e0168300, 2016.

[32] E. Tlelo-Cuautle, J. J. Rangel-Magdaleno, and L. G. de la Fraga, Engineering Applications of FPGAs, Springer, Berlin, Germany, 2016.

[33] E. Tlelo-Cuautle, J. J. Rangel-Magdaleno, A. D. Pano-Azucena, P. J. Obeso-Rodelo, and J. C. Nunez-Perez, "FPGA realization of multi-scroll chaotic oscillators," Communication in Nonlinear Science and Numerical Simulation, vol. 27, no. 1-3, pp. 66-80, 2015.

[34] O. Guillén-Fernández, M. F. Moreno-López, and E. TleloCuautle, "Issues on applying one- and multi-step numerical methods to chaotic oscillators for FPGA implementation," Mathematics, vol. 9, no. 2, p. 151, 2021. 